\title{
Do sexual health questions alter the public's response to lifestyle questionnaires?
}

\author{
P J Barker, R F Cooper
}

The national survey of sexual attitudes and lifestyles in the UK in 1990 used structured interviews to provide reliable, valid baseline information on sexual lifestyle in the general population. ${ }^{1}$ Postal surveys have also been used successfully in the past to survey sexual behaviour in the general population and are less costly, pro rata, than interview based surveys. ${ }^{2}$ However, we have been told that the experiences of social researchers in various organisations such as Social and Community Planning Research and Market and Opinion Research International Ltd suggest that some people find questions on sexual health offensive, and refuse to participate in postal surveys in which they are asked to respond to such questions. This often has a negative impact on response rates. The purpose of this study was to determine whether the inclusion of a question about sexual health in a postal survey designed to monitor lifestyle factors (such as diet, exercise, alcohol, and smoking) had an effect on the response rate.

\section{Methods}

Six hundred "lifestyle" questionnaires were dispatched to individuals whose names were randomly selected from the electoral registers in Solihull in the West Midlands. The questionnaires were identical in all respects except for the random inclusion of a sexual health question in one half of the total sample. People who did not respond to the questionnaire were sent a reminder letter and a second questionnaire three weeks after the initial questionnaire was dispatched. People who did not respond after being sent two questionnaires were classified as non-responders; a random sample of 50 non-responders was contacted by telephone though they were not required to answer the sexual health question.

The wording of the sexual health question was as follows: "If you have had a sexual partner in the past 12 months please indicate below the type of contraception you or your partner regularly use (you may tick more than one box)"

The following options were given: None, Rhythm (safe period) method, Pill, Condom, Femidom, Coil, Cap, Withdrawal, Vasectomy/Female

f Epidemiol Commun Health 1996;50:688
Solihull Health

Road, Solihull, West Midlands B91 3AH

J Barker

Correspondence to: Dr P J Barker.

Accepted for publication
sterilisation/Hysterectomy, Post menopause, Other - please give details, and Doesn't apply to me.

\section{Results}

After one questionnaire mailing the response was $53.3 \%$ in those sent the question $(160 /$ 300 ) and $51.0 \%$ in those not sent the question (153/300), which was not statistically significantly different $\left(p=0.567 \chi^{2}=0.24\right)$. After follow up with a second questionnaire the overall response rate in the two groups was virtually identical at $70 \%$ (see table 1 for details).

There were no significant age, sex, geographical or ethnic differences between the groups responding to the two questionnaires. Only $3 \%$ of the responders $(7 / 210)$ who were sent the questionnaire including the sexual health question failed to answer that particular question.

The results show that there was no significant difference in response between those who received a questionnaire including a question on sexual health and those who did not.

\section{Discussion}

Although it was not the most searching of questions on sexual health since it did not include the more sensitive aspects of sexual behaviour, the study has given the authors more confidence that sexual health questions can be included in lifestyle studies on the British public without adversely affecting the response rate.

Asking survey questions about sensitive issues has to match the prevailing cultural attitudes in society towards those issues and has always posed a dilemma for social researchers. The general household survey, for example, has only included questions on cohabitation and contraception since $1979 .^{3}$ These questions are now considered routine, yet the original decision to include them was, we are told, viewed with considerable apprehension by researchers because of the potential for an adverse effect on the response rate.

Is a similar culture shift about to take place, where the British public feels similarly able to answer questions about sexual behaviour in the context of postal surveys to assess lifestyle?

It is recommended that future studies assess the effect on response to postal surveys of including more detailed questions on sexual health.

1 Johnson AM, Wadsworth J, Wellings K, Field J, Bradshaw S. Sexual attitudes and lifestyles. Oxford: Blackwell Scientific Publications, 1994.

2 Sundet JM, Kvalem IL, Magnus P, Bakketeig LS. Prevalence of risk prone sexual behaviour in the general population of Norway. Proceedings of the Global Impact of AIDS Conference, London 1988. Abstract 4.

3 Office of Population Censuses and Surveys. General household survey. London: HMSO, 1992:198.

\begin{tabular}{llll} 
Table 1 & Overall responses to the questionnaires with and without the sexual health question \\
\hline Group & \multicolumn{2}{c}{ Sexual health question } & Total \\
\cline { 2 - 4 } & Yes & No & \\
\hline Responders & $210(70.0 \%)$ & $209(69.7 \%)$ & $419(69.8 \%)$ \\
Non-responders & 90 & 91 & 181 \\
Total & 300 & 300 & 600 \\
\hline
\end{tabular}

